\title{
EXCITED STATE ABSORPTION AND THERMOLUMINESCENCE IN Ce AND Mg DOPED YTTRIUM ALUMINUM GARNET*
}

\author{
K. WiŚNIEWSKI ${ }^{a}$, Cz. KOEPKE ${ }^{a}$, A.J. WoJTOWICZ ${ }^{a}$, W. DROZDOWSKI ${ }^{a}$, \\ M. Grinberg ${ }^{b}$, S.M. KaCZMAReK ${ }^{c}$ and J. KisIeleWski ${ }^{d}$ \\ ${ }^{a}$ Institute of Physics, Nicholas Copernicus University \\ Grudziądzka 5/7, 87-100 Toruń, Poland \\ ${ }^{b}$ Institute of Experimental Physics, Gdańsk University \\ Wita Stwosza 57, 80-952 Gdańsk, Poland \\ 'Institute of Optoelectronics, Military University of Technology, 01-489 Warsaw, Poland \\ ${ }^{d}$ Institute of Electronic Materials Technology, 01-919 Warsaw, Poland
}

(Received September 16, 1998; in final form January 29, 1999)

In this paper we report preliminary results of optical studies on $\mathrm{Y}_{3} \mathrm{Al}_{5} \mathrm{O}_{12}$ (YAG) crystals codoped with $\mathrm{Ce}$ and $\mathrm{Mg}$. By using measurements of luminescence, absorption, and luminescence excitation spectra we demonstrate that although the basic features introduced to the YAG host by the Ce-doping remain intact, the $\mathrm{Mg}$-codoping imposes some significant changes on other properties of the material. These changes are potentially important for laser and/or scintillator applications of YAG:Ce and are due, most likely, to modifications of defect populations in the material. We characterize them by using the techniques of thermoluminescence and excited state absorption under excimer laser pumping. These techniques, interestingly, yield results that seem inconsistent. While the thermoluminescence signal of the Mg-doped sample is strongly reduced, suggesting that trap concentrations in the presence of $\mathrm{Mg}$ are suppressed, the excited state absorption signal, which we also relate to the traps, is higher. We offer a tentative explanation of this contradiction between the two experiments that involves a massive transfer of electrons from the Mg-related defects to the excited state absorption centers caused by the excimer pump itself.

PACS numbers: 78.55.Hx, 78.55.-m, 78.60.Kn, 73.50.Gr, 29.40.Mc, 78.20.-e, 78.20.Wc, 78.40.-q,

*The results of this paper were initially presented at The Jabtoniski Centennial Conference on Luminescence and Photophysics, July 23-27, 1998, Toruń, Poland.

†e-mail: krzys@phys.uni.torun.pl 


\section{Introduction}

Optical properties of Ce-doped $\mathrm{Y}_{3} \mathrm{Al}_{5} \mathrm{O}_{12}$ (YAG) have been studied and published previously [1-4]. The fast, efficient and broad band emission peaking at about $525 \mathrm{~nm}$ in YAG:Ce is due to the $5 d-4 f$ transitions of $\mathrm{Ce}^{3+}$ ions. The high quantum efficiency, the width of the emission band, the relatively large Stokes shift and the split ground state make the material potentially useful in solid state tunable laser and scintillator applications [1].

Unfortunately, it has been established that YAG:Ce does not show the net gain in the potentially attractive range of wavelengths because of the excited state absorption (ESA). The reported ESA spectra of YAG:Ce cover all of that range as reported in Refs. [1-4]. The strength and breadth of the ESA spectrum allegedly disqualify the material as an active medium of the solid state tunable laser [3, 4].

We note, however, that in the ESA spectra reported previously (Refs. [1-4], see also Fig. 4) the characteristic Ce-related band is situated at the wavelengths almost entirely above the long wavelength limit of the $\mathrm{Ce}^{3+}$ emission band. On the other hand, the featureless and flat component that actually does overlap the $\mathrm{Ce}^{3+}$ emission band, may well not be intrinsic to $\mathrm{Ce}$ and may, instead, originate in various defects characteristic of the YAG host as suggested before [5-7]. Since concentrations of defects can be manipulated in many ways (doping with aliovalent ions, annealing in different atmospheres and so on) it may well be that the ESA in the critical range of wavelengths can also be modified and, hopefully, reduced.

In this paper we report some preliminary results on YAG:Ce that was modified by codoping with the aliovalent dopant, $\mathrm{Mg}$, in amount comparable to that of the Ce-activator. In general we expect that the introduction of the acceptor-like ion to the YAG host will either strip the electrons from all the defects with levels positioned at the upper part of the forbidden energy gap or, by creating the defect-ion association, it will transform those defects into deeper ones. In either case the change in the ESA is likely to be beneficial for the laser operation of the material; either the defect is rendered optically inactive or its absorption is shifted away from the spectral range of interest.

\section{Materials and experiments}

The two crystals we studied, YAG:0.2\%Ce and YAG:0.2\%Ce, $0.1 \% \mathrm{Mg}$, were grown at the Institute of Electronic Materials Technology using the Czochralski method. The following oxides: $\mathrm{Y}_{2} \mathrm{O}_{3}, \mathrm{Al}_{2} \mathrm{O}_{3}, \mathrm{CeO}_{2}$ and $\mathrm{MgO}$, were used as starting materials. The composition of the mixture was chosen to conform to the following formula [8]:

$$
\begin{aligned}
& 3(1-x-y) \mathrm{Y}_{2} \mathrm{O}_{3}+6 x \mathrm{CeO}_{2}+6 y \mathrm{MgO}-3(x-y) \mathrm{O}_{2}+5 \mathrm{Al}_{2} \mathrm{O}_{3} \\
& \rightarrow 2 \mathrm{Y}_{3(1-x-y)} \mathrm{Ce}_{3 x} \mathrm{Mg}_{3 y} \mathrm{Al}_{5} \mathrm{O}_{12 \pm \sigma}
\end{aligned}
$$

where $x$ and $y$ are the expected $\mathrm{Mg}$ and Ce concentration values in the final product, that is the crystal of YAG:Ce,Mg.

The experimental setups that were used for absorption, emission, and luminescence excitation spectra are fairly typical and as such need not to be described here. The ESA spectra were measured using an experimental setup described in 
Ref. [9]. The results are expressed as the excited state transmission, $T_{\mathrm{ES}}$, represented by the following ratio, $I_{\mathrm{p}} / I_{\mathrm{u}}$, where $I_{\mathrm{p}}$ is the intensity of the probe beam that passed the pumped sample and $I_{\mathrm{u}}$ is the intensity of the probe beam that passed the unpumped sample. All ESA measurements were conducted at room temperature. The glow curves were recorded between 300 and $600 \mathrm{~K}$ using a RISO TL/OSL system, model TL/OSL-DA-12 [10], at the three heating rates of 1,2, and $5 \mathrm{~K} / \mathrm{s}$. Before the thermoluminescence (TL) runs, the samples have been exposed to $196 \mathrm{keV} \beta$-rays from an Sr/Y-90 radioactive source for $2 \mathrm{~s}$. The TL glow curves were taken with the sample in the Ar atmosphere. The interference filter HA3 was used to reject any undesirable emissions (mostly thermal radiation from the sample and the heating element).

\section{Results}

In Fig. 1 we compare absorption spectra of YAG:Ce and YAG:Ce,Mg. Both spectra show two bands, at $450 \mathrm{~nm}$ and $340 \mathrm{~nm}$, due to $4 f \rightarrow 5 d$ transitions involving the two lowest energy $d$-levels of the $\mathrm{Ce}^{3+}$ ion in the YAG crystal. The third band, at $230 \mathrm{~nm}$, clearly visible in YAG:Ce, disappears in YAG:Ce, $\mathrm{Mg}$, probably buried under steeply rising absorption that was absent in the $\mathrm{Mg}$-free sample. This feature is clearly due to the $\mathrm{Mg}$ or $\mathrm{Mg}$-related defect in the YAG host and can be associated with a level some $3.5 \mathrm{eV}$ below the conduction band. This estimate puts this $\mathrm{Mg}$-related level in the vicinity of the level associated with the $\mathrm{Ce}^{3+}$ in the ground state [11].

The excitation spectra of the emission from the two samples, YAG:Ce and YAG:Ce, $\mathrm{Mg}$, were measured with the receiving monochromator set at $530 \mathrm{~nm}$.

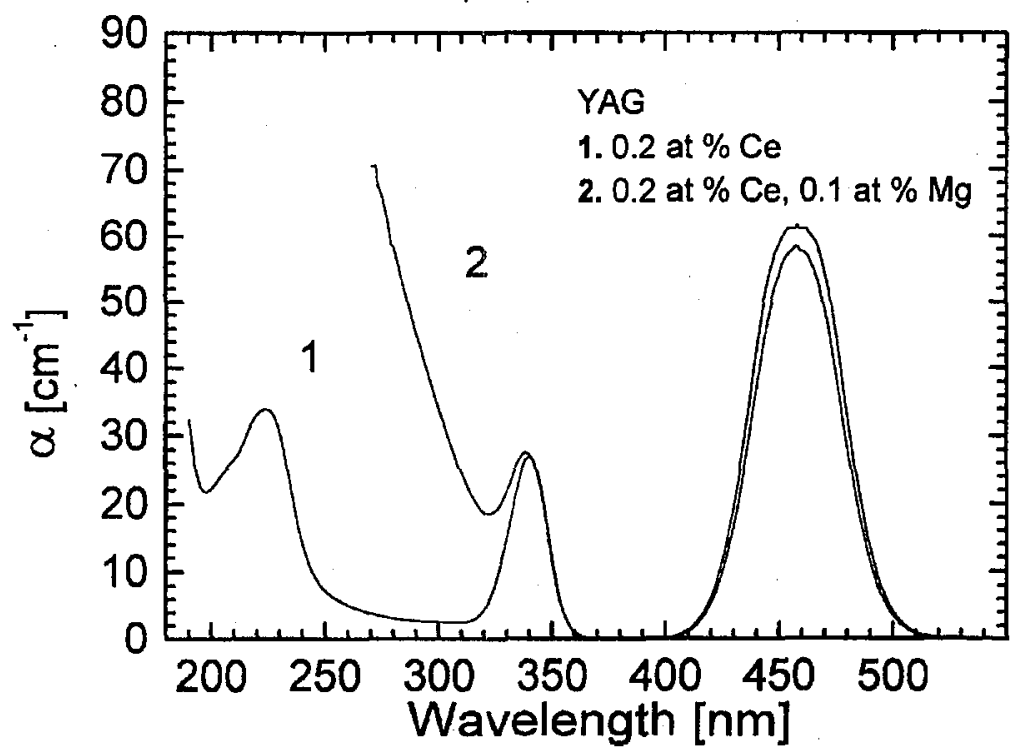

Fig. 1. Absorption spectra of YAG:Ce and YAG:Ce,Mg. 


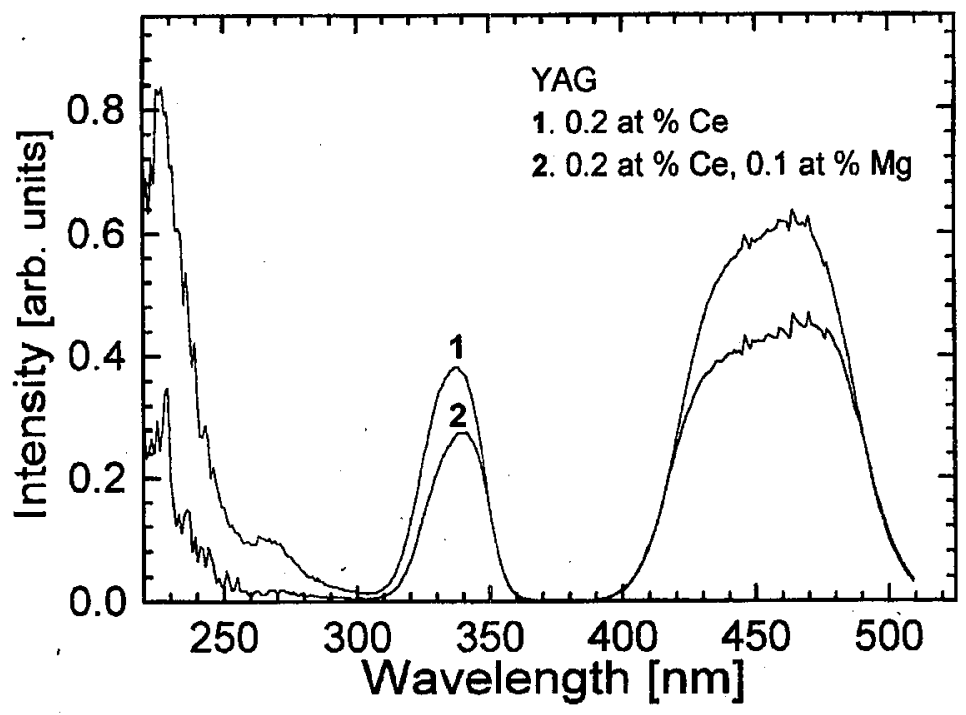

Fig. 2. Excitation spectra of YAG:Ce and YAG:Ce,Mg detected at $\lambda_{\text {emi }}=530 \mathrm{~nm}$.

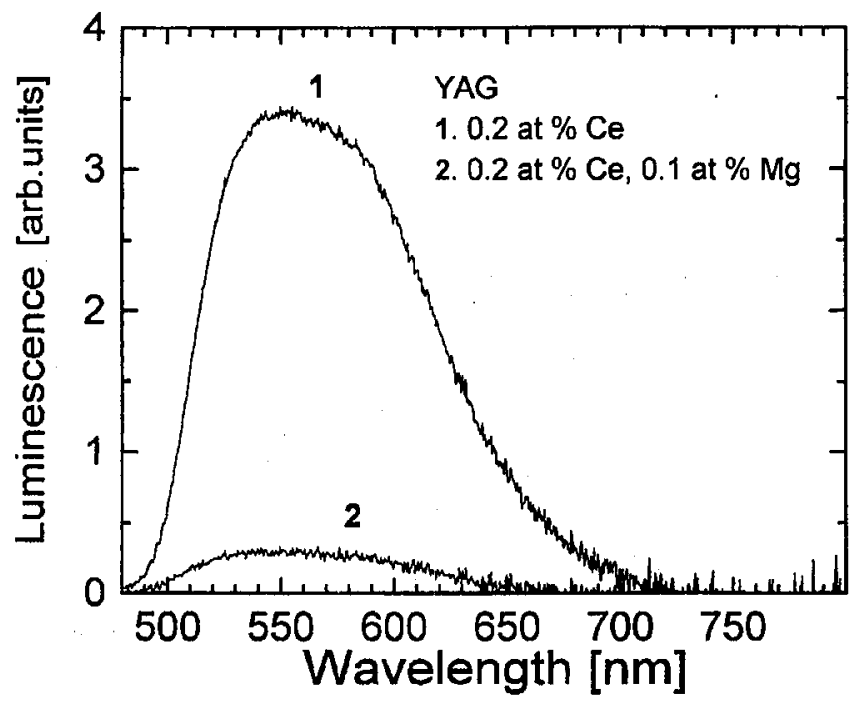

Fig. 3. Luminescence spectra of YAG:Ce and YAG:Ce,Mg detected with excitation: $\lambda_{\text {exc }}=308 \mathrm{~nm}$.

These spectra are presented in Fig. 2. The three resolved bands are identical to those observed in the absorption spectra. The relatively lower efficiency of $\mathrm{Ce}^{3+}$ excitation below $300 \mathrm{~nm}$ must be due to the $\mathrm{Mg}$-related absorption that does not lead to $\mathrm{Ce}$ emission. Some differences in relative intensities of corresponding excitation bands in the two samples might be caused by the $\mathrm{Mg}$-induced $\mathrm{Ce}^{3+}-\mathrm{Ce}^{4+}$ conversion, although the spectra are distorted (compare Fig. 1) and any meaningful comparison is difficult. 
In Fig. 3 we present the emission spectra of the two YAG samples. At the excitation wavelength of $308 \mathrm{~nm}$ the competition from the $\mathrm{Mg}$-related absorption strongly reduces the efficiency of Ce excitation and, consequently, the height of the spectrum shown by trace (2).

The results of the ESA measurements, along with the previously measured values of Miniscalco et al. [1] (circles) and Hamilton et al. [4] (crosses), are shown in Fig. 4. Note that as the literature values are expressed differently than ours they are not directly comparable. The pump beam from the excimer laser had a wavelength of $308 \mathrm{~nm}$ that clearly falls below the Ce-related photoconductivity threshold [11] and well into the Mg-related absorption shown in Fig. 1. In the two $T_{\mathrm{ES}}$ spectra of YAG:Ce and YAG:Ce, $\mathrm{Mg}$, shown in the figure by solid lines and labeled accordingly, we have omitted the range of wavelengths between 400 and $500 \mathrm{~nm}$. In this range the meaningful measurement is hardly possible because of the strong interference from the ground state absorption bleaching $[9,12]$, due to a very large absorption coefficient (see Fig. 1). Clearly, the YAG:Ce spectrum closely resembles the literature curve shown by crosses and circles, with the characteristic tail on the high energy side assigned to the transient color centers as discussed before. Those centers are known to occur in YAG and many other materials although usually they are activated by a much shorter wavelength excitation [7].

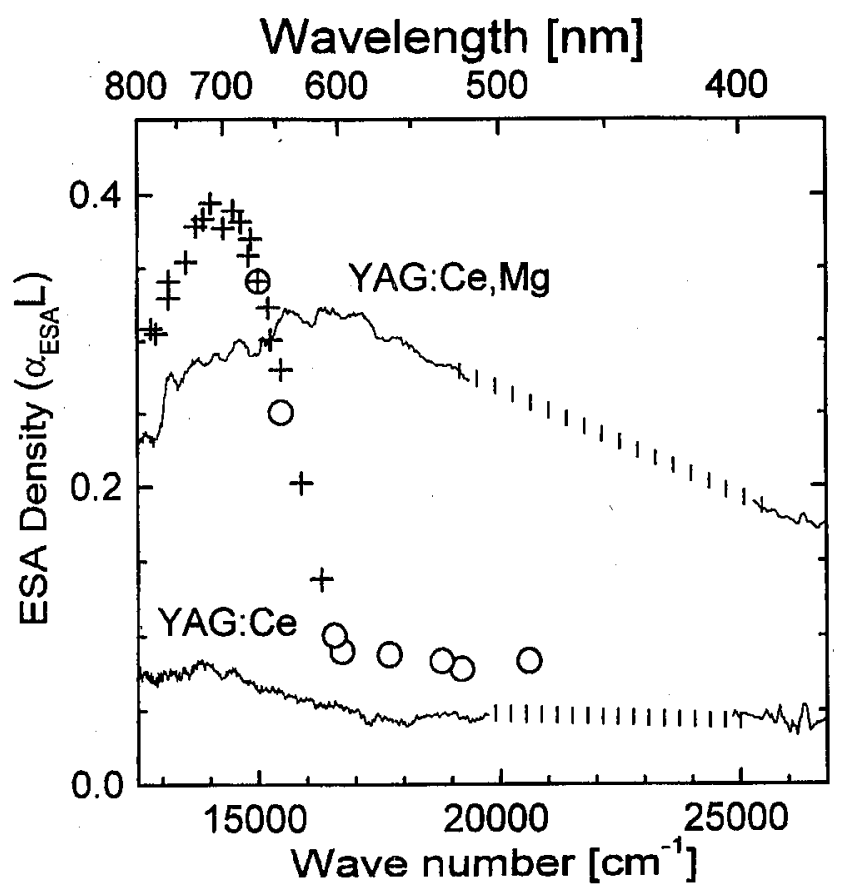

Fig. 4. Exited state absorption spectra of YAG:Ce and YAG:Ce,Mg. The spectrum with circles and crosses is the ESA spectrum of YAG:Ce taken from [4]. The fragments of vertical bars are inserted artificially in the regions where the ESA spectra were severely distorted by the ground state absorption bleaching. 
This difference in wavelengths is possibly due to the effectively lower bandgap induced by $\mathrm{Mg}$-doping of YAG.

On the other hand the YAG:Ce,Mg spectrum, also shown in Fig. 4, is very different, with a much higher intensity, a shifted maximum and a different shape. It goes without saying that this spectrum is not due to Ce ions. It rather must reflect the difference between the two samples created by the Mg-codoping and, possibly, to the increased population of some traps.

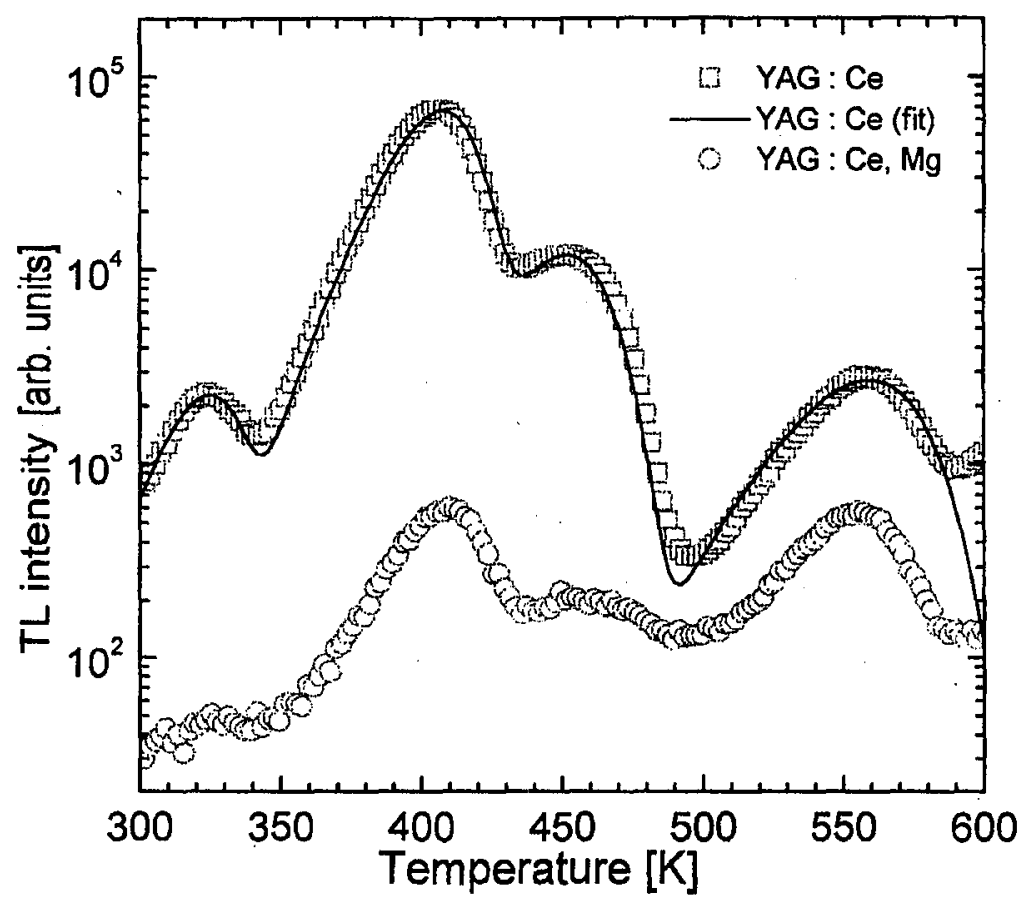

Fig. 5. The glow curves of YAG:Ce and YAG:Ce,Mg crystals.

To explore traps in YAG:Ce and YAG:Ce, $\mathrm{Mg}$, we measured thermoluminescence in both materials. The measured glow curves of the two samples are shown in Fig. 5. At the heating rate of $1 \mathrm{~K} / \mathrm{s}$ there are four peaks at about 330, 410, 460, and $550 \mathrm{~K}$. The glow curves recorded at the heating rates of 2 and $5 \mathrm{~K} / \mathrm{s}$ are very similar but, as expected, have their maxima shifted to higher temperatures. In the frame of a simple model of Randall and Wilkins [13], the peaks are associated with the four different electron traps. To find the trap parameters we used the Randall-Wilkins formula

$$
I(T)=\sum_{i=1}^{4} n_{0 i} s_{i} \exp \left(-\frac{E_{i}}{k_{\mathrm{B}} T}\right) \exp \left(-\frac{S_{i}}{\beta} \int_{T_{0}}^{T} \exp \left(-\frac{E_{i}}{k_{\mathrm{B}} T}\right) \mathrm{d} T\right),
$$

where $I(T)$ is the TL intensity at the temperature $T, \beta$ is the heating rate, $n_{0}$ - the initial concentration of filled traps, $E$ - the trap depth, $s$ - the frequency factor, and $k_{\mathrm{B}}$ is the Boltzmann constant. This expression was fitted to 
the experimental points for YAG:Ce. A more detailed description of the fitting procedure is given in Ref. [14]. The results of the fit are shown by a solid line in Fig. 5, and are summarized in Table. Interestingly, the much weaker glow curves of YAG:Ce, $\mathrm{Mg}$ can be well reproduced using the same sets of trap parameters. Obviously, $\mathrm{Mg}$-doping does not create any new traps glowing in the range of tem-

\section{TABLE}

The depths and frequency factors of the traps in YAG:Ce and YAG: $\mathrm{Ce}, \mathrm{Mg}$ crystals.

\begin{tabular}{c|c|c}
\hline \hline Trap & $E[\mathrm{eV}]$ & $\ln s$ \\
\hline 1 & 0.70 & 22.6 \\
2 & 1.01 & 26.2 \\
3 & 1.01 & 23.1 \\
4 & 1.20 & 21.8
\end{tabular}

peratures between 300 and $600 \mathrm{~K}$. Contrary to the ESA results the TL signal is much lower suggesting that traps, particularly the one glowing at about $410 \mathrm{~K}$, are suppressed. Since the relative concentrations of various traps are also changed the effect cannot be due exclusively to competition for holes between the Ce ions and the $\mathrm{Mg}$-related defect responsible for the electron-filled level $3.5 \mathrm{eV}$ below the conduction band, identified previously.

\section{Discussion}

To resolve the apparent contradiction between the results of the two experiments, ESA and TL, we note that the excimer laser pump at the wavelength of $308 \mathrm{~nm}$ is absorbed mostly by the Mg-related defects identified previously. Only a relatively small fraction of the pump beam intensity is used to excite the Ce ions. As a consequence while the population of the excited $\mathrm{Ce}^{3+}$ ions stays low there is a relatively large population of electrons in the conduction band coming from the Mg-related defects that absorb most of the excimer light. These electrons are then free to be captured by various defect centers (traps), including those responsible for the ESA. Therefore, even with the Mg-doping effectively quenching some defects (which explains the results of the TL measurements), the coincidence between the excimer pump wavelength and the Mg-related absorption is responsible for effectively transferring electrons from the $\mathrm{Mg}$-related defects to some defect centers (traps) responsible for the transient absorption (ESA). Since in the absence of $\mathrm{Mg}$ no such defects exist, most of the excimer light is absorbed by Ce ions and no electrons are transferred to the empty traps. The ESA spectrum is then due to Ce ions and not to the traps. A possible model describing such processes is presented in Fig. 6 (see also Ref. [15]).

Since we have the thermal activation energies (Table) and the ESA maximum energy, we can use the simple configuration coordinate diagram to represent both 


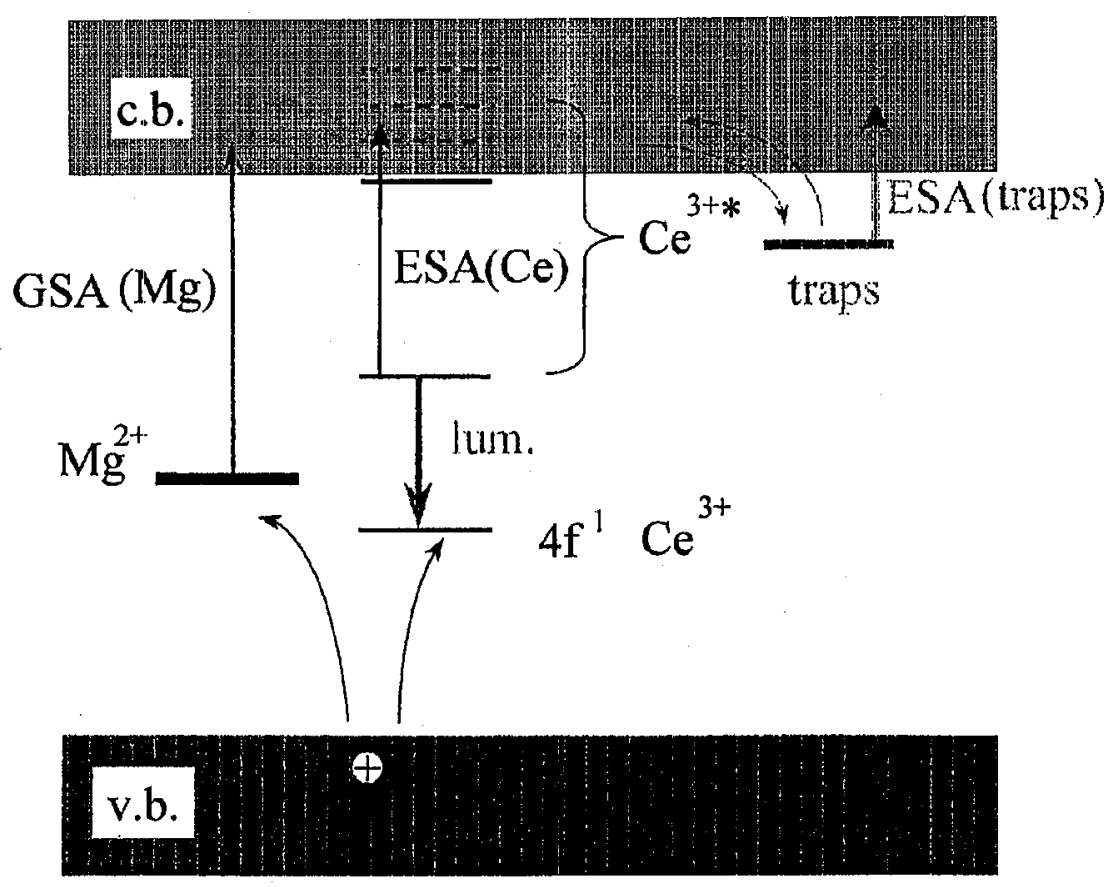

Fig. 6. Model of the ESA for YAG:Ce crystal codoped with Mg.

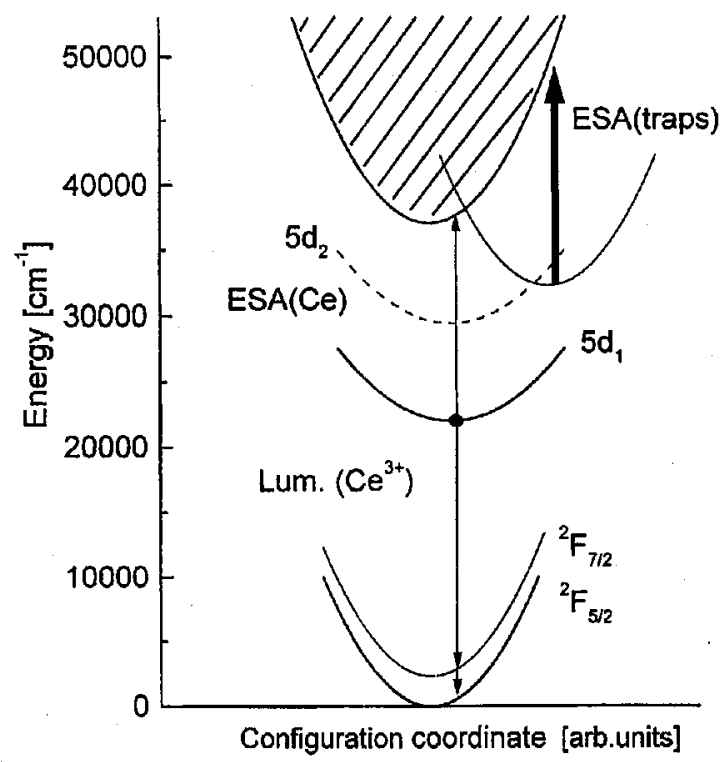

Fig. 7. Simple configuration coordinate diagram illustrating the formation of both ESA components. 
the Ce ion and the trap. Such a diagram is presented in Fig. 7 for the trap of $1.2 \mathrm{eV}$ energy depth. Note that the amount of the electron-lattice coupling is larger for the shallower trap and that the coupling for the $1.2 \mathrm{eV}$ trap is already quite large. The conclusion must be, therefore, that the trap actually responsible for the observed ESA may be even deeper than $1.2 \mathrm{eV}$.

\section{Conclusions}

There are two contradictory observations associated with codoping of YAG:Ce crystals with the $\mathrm{Mg}^{2+}$ ions, the increased ESA and the reduced TL intensity. It appears that although the $\mathrm{Mg}$-codoping induces gross changes in defect concentrations in YAG crystals, as expected, those changes do not translate into reduced ESA, as desired. On the contrary, the ESA from the YAG:Ce,Mg crystal is much higher. The tentative explanation we offer now relies on the accidental coincidence between the $308 \mathrm{~nm}$ pump light from the excimer laser and the absorption band introduced by the $\mathrm{Mg}$-related defect. The excimer light is, therefore, absorbed by those defects, not by Ce ions. This, in turn, causes an efficient transfer of electrons from the $\mathrm{Mg}$-related defects to the centers responsible for the transient absorption (ESA). More experiments are planned to resolve this problem.

\section{Acknowledgments}

This work was partially sponsored by the Committee for Scientific Research Poland (projects no. 2 P03B 00313 and 2 P03B 049 14), and by the local University grant (UMK no. 363-F, 1998).

\section{References}

[1] W.J. Miniscalco, J.M. Pellegrino, W.M. Yen, J. Appl. Phys. 49, 6109 (1978).

[2] R.R. Jacobs, W.F. Krupke, M.J. Weber, Appl. Phys. Lett. 33, 410 (1978).

[3] D.S. Hamilton, in: Proc. First Intern. Conf. Tunable Solid State Lasers, La Jolla (California) 1984, Eds. P. Hammerling, A.B Budgov, A. Pinto, Springer-Verlag, Berlin 1985, p. 80.

[4] D.S. Hamilton, S.K. Gayen, G.J. Pogatshnik, R.D. Ghen, W.J. Miniscalco, Phys. Rev. B 39, 8807 (1989).

[5] R.J. Landry, E. Snitzer, R.H. Bartram, J. Appl. Phys. 42, 3827 (1971).

[6] R.S. Crandall, M. Mikkor, Phys. Rev. A 1247, 138 (1964).

[7] K.S. Bagdasarov, L.B. Pasternak, B.K. Sevastyanov, Sov. J. Quantum Electron. 7, 965 (1977).

[8] S.M. Kaczmarek, D.J. Sugak, A.O. Matkovskii, Z. Moroz, M. Kwaśny, A.N. Durygin, Nucl. Instrum. Methods B 132, 647 (1997).

[9] Cz. Koepke, K. Wiśniewski, M. Grinberg, D.L. Russell, K. Holliday, G.H. Beall, J. Lumin. 78, 1234 (1998).

[10] L. Botter-Jensen, Nucl. Tracks Radiat. Meas. 14, 177 (1988).

[11] C. Pedrini, F. Rogemond, D.S. McClure, J. Appl. Phys. 59, 1196 (1986).

[12] B. Di Bartolo, Optical Interaction in Solids, Wiley, New York 1968.

[13] J.T. Randall, M.H.F. Wilkins, Proc. R. Soc. Lond. A 184, 366 (1945). 
[14] W. Drozdowski, K.R. Przegiętka, A.J. Wojtowicz, H.L. Oczkowski, Acta Phys. Pol. A 95, 251 (1999).

[15] J. Barzowska, A. Kubicki, M. Grinberg, S. Kaczmarek, Z. Euczyński, A.J. Wojtowicz, Cz. Koepke, Acta Phys. Pol. A 95, 395 (1999). 